\title{
Aerodynamic-Free Adaptive Control of the NASA Generic Transport Model
}

\author{
Frantisek M. Sobolic* and Dennis S. Bernstein ${ }^{\dagger}$ \\ University of Michigan, 1320 Beal Avenue Ann Arbor, MI 48109-2140
}

\begin{abstract}
Unanticipated and unknown changes in an aircraft's aerodynamic stability derivatives may cause undesirable effects that render pre-designed control laws unsuitable for maintaining stability. Gradual changes in the stability derivatives may be due to icing on lifting surfaces, whereas structural damage may cause a sudden change. For this study, we use the nonlinear NASA Generic Transport Model (GTM) to investigate the ability of retrospective cost adaptive control (RCAC) to compensate for changes in the stability derivatives while maintaining steady level flight despite unknown variations in the aerodynamic modeling information.
\end{abstract}

\section{Introduction}

Aircraft control under emergency conditions poses severe challenges. For example, control surface faults may limit the maneuverability of the aircraft and require unconventional control strategies $[2,5,6,9]$. Although anticipated faults can be compensated for by contingency plans, unexpected faults require real-time adaptation under unknown conditions.

In the present paper we are concerned with unanticipated and unknown changes in the aerodynamics of the aircraft as modeled by changes in its stability derivatives [11]. For each airspeed and altitude, stability derivatives provide a linearized approximation of the aerodynamic forces and moments on the aircraft as functions of perturbations from steady flight conditions. For aircraft certification and autopilot development, stability derivatives are typically determined through computational techniques and wind tunnel testing. These data can be stored in a lookup table for simulation studies.

In the present paper we consider emergency flight under abruptly or gradually changing stability derivatives. In particular, we apply retrospective cost adaptive control (RCAC) to various scenarios, such as slowly changing lift and drag coefficients to emulate the effect of icing. Of particular interest is the evolution of the RCAC controller gains in response to unknown changes in the aircraft dynamics.

RCAC has been developed in $[3,7,8,12,13]$ and applied to aircraft flight control in [4]. As in [4], we use the NASA Generic Transport Model $[1,10]$ to investigate the ability of RCAC to compensate for changing stability derivatives. To this end, we modify the aerodynamic database in the GTM. All simulations of RCAC are carried out on the fully nonlinear model, which accesses the aerodynamic database at each time step of the simulation.

In Section II of the paper, we present the RCAC algorithm from $[3,7,8,12,13]$ for a general multi-input, multi-output discrete-time system. This section defines the retrospective cost used to update the control law based on previous performance. In Section III, we describe how the stability derivatives are accessed and modified in the Simulink GTM, and we present the control architecture used for RCAC. We also define the performance variables and tuning parameters used for the examples presented in Section IV. Finally, in Section $\mathrm{V}$ we summarize our findings with conclusions.

\footnotetext{
*Graduate Student, Department of Aerospace Engineering, fsobolic@umich.edu. Member AIAA.

†Professor, Department of Aerospace Engineering, dsbaero@umich.edu. Member AIAA.
} 


\section{Adaptive Controller Design}

\section{II.A. Problem Formulation}

Consider the MIMO discrete-time system

$$
\begin{aligned}
x(k+1) & =A x(k)+B u(k)+D_{1} w(k), \\
y(k) & =C x(k)+D_{2} w(k), \\
z(k) & =E_{1} x(k)+E_{0} w(k),
\end{aligned}
$$

where $x(k) \in \mathbb{R}^{n}, y(k) \in \mathbb{R}^{l_{y}}, z(k) \in \mathbb{R}^{l_{z}}, u(k) \in \mathbb{R}^{l_{u}}, w(k) \in \mathbb{R}^{l_{w}}$, and $k \geq 0$. Our goal is to develop an adaptive output feedback controller that minimizes the performance variable $z$ in the presence of the exogenous signal $w$ with minimal modeling information about the dynamics and $w$. Note that $w$ can represent either a command signal to be followed, an external disturbance to be rejected, or both. The system (1)-(3) can represent a sampled-data application arising from a continuous-time system with sample and hold operations.

If $D_{1}=0$ and $E_{0} \neq 0$, then the objective is to have the output $E_{1} x$ follow the command signal $-E_{0} w$. On the other hand, if $D_{1} \neq 0$ and $E_{0}=0$, then the objective is to reject the disturbance $w$ from the performance measurement $E_{1} x$. Furthermore, if $D_{1}=\left[\begin{array}{ll}\hat{D}_{1} & 0\end{array}\right], E_{0}=\left[\begin{array}{ll}0 & \hat{E}_{0}\end{array}\right]$, and $w(k)=\left[\begin{array}{l}w_{1}(k)^{\mathrm{T}} w_{2}(k)^{\mathrm{T}}\end{array}\right]^{\mathrm{T}}$, then the objective is to have $E_{1} x$ follow the command $-\hat{E}_{0} w_{2}$ while rejecting the disturbance $w_{1}$. Lastly, if $D_{1}$ and $E_{0}$ are empty matrices, then the objective is output stabilization, that is, convergence of $z$ to zero.

\section{II.B. Retrospective Cost}

For $i \geq 1$, define the Markov parameter of $G_{z u}$ given by

$$
H_{i} \triangleq E_{1} A^{i-1} B
$$

For example, $H_{1}=E_{1} B$ and $H_{2}=E_{1} A B$. Let $r$ be a positive integer. Then, for all $k \geq r$,

$$
\begin{aligned}
x(k)=A^{r} x(k-r) & +\sum_{i=1}^{r} A^{i-1} B u(k-i) \\
& +\sum_{i=1}^{r} A^{i-1} D_{1} w(k-i),
\end{aligned}
$$

and thus

$$
\begin{aligned}
z(k)=E_{1} A^{r} x(k-r) & +\sum_{i=1}^{r} E_{1} A^{i-1} D_{1} w(k-i) \\
& +E_{0} w(k)+\bar{H} \bar{U}(k-1),
\end{aligned}
$$

where

$$
\bar{H} \triangleq\left[\begin{array}{lll}
H_{1} & \cdots & H_{r}
\end{array}\right] \in \mathbb{R}^{l_{z} \times r l_{u}}
$$

and

$$
\bar{U}(k-1) \triangleq\left[\begin{array}{c}
u(k-1) \\
\vdots \\
u(k-r)
\end{array}\right] .
$$

Next, we rearrange the columns of $\bar{H}$ and the components of $\bar{U}(k-1)$ and partition the resulting matrix and vector so that

$$
\bar{H} \bar{U}(k-1)=\mathcal{H}^{\prime} U^{\prime}(k-1)+\mathcal{H} U(k-1),
$$


where $\mathcal{H}^{\prime} \in \mathbb{R}^{l_{z} \times\left(r l_{u}-l_{U}\right)}, \mathcal{H} \in \mathbb{R}^{l_{z} \times l_{U}}, U^{\prime}(k-1) \in \mathbb{R}^{r l_{u}-l_{U}}$, and $U(k-1) \in \mathbb{R}^{l_{U}}$. Then, we can rewrite (6) as

$$
z(k)=\mathcal{S}(k)+\mathcal{H} U(k-1),
$$

where

$$
\mathcal{S}(k) \triangleq E_{1} A^{r} x(k-r)+\sum_{i=1}^{r} E_{1} A^{i-1} D_{1} w(k-i)+E_{0} w(k)+\mathcal{H}^{\prime} U^{\prime}(k-1) .
$$

Next, for $j=1, \ldots, s$, we rewrite (8) with a delay of $k_{j}$ time steps, where $0 \leq k_{1} \leq k_{2} \leq \cdots \leq k_{s}$, in the form

$$
z\left(k-k_{j}\right)=\mathcal{S}_{j}\left(k-k_{j}\right)+\mathcal{H}_{j} U_{j}\left(k-k_{j}-1\right),
$$

where (9) becomes

$$
\mathcal{S}_{j}\left(k-k_{j}\right) \triangleq E_{1} A^{r} x\left(k-k_{j}-r\right)+\sum_{i=1}^{r} E_{1} A^{i-1} D_{1} w\left(k-k_{j}-i\right)+E_{0} w\left(k-k_{j}\right)+\mathcal{H}_{j}^{\prime} U_{j}^{\prime}\left(k-k_{j}-1\right)
$$

and (7) becomes

$$
\bar{H} \bar{U}\left(k-k_{j}-1\right)=\mathcal{H}_{j}^{\prime} U_{j}^{\prime}\left(k-k_{j}-1\right)+\mathcal{H}_{j} U_{j}\left(k-k_{j}-1\right),
$$

where $\mathcal{H}_{j}^{\prime} \in \mathbb{R}^{l_{z} \times\left(r l_{u}-l_{U_{j}}\right)}, \mathcal{H}_{j} \in \mathbb{R}^{l_{z} \times l_{U_{j}}}, U_{j}^{\prime}\left(k-k_{j}-1\right) \in \mathbb{R}^{r l_{u}-l_{U_{j}}}$, and $U_{j}\left(k-k_{j}-1\right) \in \mathbb{R}^{l_{U_{j}}}$. Now, by stacking $z\left(k-k_{1}\right), \ldots, z\left(k-k_{s}\right)$, we define the extended performance

$$
Z(k) \triangleq\left[\begin{array}{c}
z\left(k-k_{1}\right) \\
\vdots \\
z\left(k-k_{s}\right)
\end{array}\right] \in \mathbb{R}^{s l_{z}} .
$$

Therefore,

$$
Z(k) \triangleq \tilde{\mathcal{S}}(k)+\tilde{\mathcal{H}} \tilde{U}(k-1)
$$

where

$$
\tilde{\mathcal{S}}(k) \triangleq\left[\begin{array}{c}
\mathcal{S}_{1}\left(k-k_{1}\right) \\
\vdots \\
\mathcal{S}_{s}\left(k-k_{s}\right)
\end{array}\right] \in \mathbb{R}^{s l_{z}},
$$

$\tilde{U}(k-1)$ has the form

$$
\tilde{U}(k-1) \triangleq\left[\begin{array}{c}
u\left(k-q_{1}\right) \\
\vdots \\
u\left(k-q_{l_{\tilde{U}}}\right)
\end{array}\right] \in \mathbb{R}^{l_{\tilde{U}}},
$$

where, for $i=1, \ldots, l_{\tilde{U}}, k_{1} \leq q_{i} \leq k_{s}+r$, and $\tilde{\mathcal{H}} \in \mathbb{R}^{s l_{z} \times l_{\tilde{U}}}$ is constructed according to the structure of $\tilde{U}(k-1)$. The vector $\tilde{U}(k-1)$ is formed by stacking $U_{1}\left(k-k_{1}-1\right), \ldots, U_{s}\left(k-k_{s}-1\right)$ and removing copies of repeated components.

Next, we define the retrospective performance

$$
\hat{z}\left(k-k_{j}\right) \triangleq \mathcal{S}_{j}\left(k-k_{j}\right)+\mathcal{H}_{j} \hat{U}_{j}\left(k-k_{j}-1\right),
$$

where the past controls $U_{j}\left(k-k_{j}-1\right)$ in (10) are replaced by the retrospective controls $\hat{U}_{j}\left(k-k_{j}-1\right)$. In analogy with (12), the extended retrospective performance for (16) is defined as

$$
\hat{Z}(k) \triangleq\left[\begin{array}{c}
\hat{z}\left(k-k_{1}\right) \\
\vdots \\
\hat{z}\left(k-k_{s}\right)
\end{array}\right] \in \mathbb{R}^{s l_{z}}
$$


and thus is given by

$$
\hat{Z}(k)=\tilde{\mathcal{S}}(k)+\tilde{\mathcal{H}} \hat{\tilde{U}}(k-1),
$$

where the components of $\hat{\tilde{U}}(k-1) \in \mathbb{R}^{l} \tilde{U}$ are the components of $\hat{U}_{1}\left(k-k_{1}-1\right), \ldots, \hat{U}_{s}\left(k-k_{s}-1\right)$ ordered in the same way as the components of $\tilde{U}(k-1)$. Subtracting (13) from (18) yields

$$
\hat{Z}(k)=Z(k)-\tilde{\mathcal{H}} \tilde{U}(k-1)+\tilde{\mathcal{H}} \hat{\tilde{U}}(k-1) .
$$

Finally, we define the retrospective cost function

$$
J(\hat{\tilde{U}}(k-1), k) \triangleq \hat{Z}^{\mathrm{T}}(k) R(k) \hat{Z}(k),
$$

where $R(k) \in \mathbb{R}^{l_{z} s \times l_{z} s}$ is a positive-definite performance weighting. The goal is to determine refined controls $\hat{\tilde{U}}(k-1)$ that would have provided better performance than the controls $U(k)$ that were applied to the system. The refined control values $\hat{\tilde{U}}(k-1)$ are subsequently used to update the controller.

\section{II.C. Cost Function Optimization with Adaptive Regularization}

To ensure that (20) has a global minimizer, we consider the regularized cost

$$
\bar{J}(\hat{\tilde{U}}(k-1), k) \triangleq \hat{Z}^{\mathrm{T}}(k) R_{Z} \hat{Z}(k)+\hat{\tilde{U}}^{\mathrm{T}}(k-1) R_{U} \hat{\tilde{U}}(k-1),
$$

where $R_{Z} \in \mathbb{R}^{\hat{Z}} \geq 0$, and $R_{U} \in \mathbb{R}^{\hat{U}} \geq 0$. Substituting (19) into (21) yields

$$
\bar{J}(\hat{\tilde{U}}(k-1), k)=\hat{\tilde{U}}(k-1)^{\mathrm{T}} \mathcal{A}(k) \hat{\tilde{U}}(k-1)+\hat{\tilde{U}}^{\mathrm{T}}(k-1) \mathcal{B}^{\mathrm{T}}(k)+\mathcal{C}(k),
$$

where

$$
\begin{aligned}
& \mathcal{A}(k) \triangleq \tilde{\mathcal{H}}^{\mathrm{T}} R_{Z} \tilde{\mathcal{H}}+R_{U}, \\
& \mathcal{B}(k) \triangleq 2 \tilde{\mathcal{H}}^{\mathrm{T}} R_{Z}[Z(k)-\tilde{\mathcal{H}} \tilde{U}(k-1)], \\
& \mathcal{C}(k) \triangleq Z^{\mathrm{T}}(k) R_{Z} Z(k)-2 Z^{\mathrm{T}}(k) R_{Z} \tilde{\mathcal{H}} \tilde{U}(k-1)+\tilde{U}^{\mathrm{T}}(k-1) \tilde{\mathcal{H}}^{\mathrm{T}} R_{Z} \tilde{\mathcal{H}} \tilde{U}(k-1) .
\end{aligned}
$$

If either $\tilde{\mathcal{H}}$ has full column rank or $R_{Z}>0$ and $R_{U}>0$, then $\mathcal{A}(k)$ is positive definite. In this case, $\bar{J}(\hat{\tilde{U}}(k-1), k)$ has the unique global minimizer

$$
\hat{\tilde{U}}(k-1)=-\frac{1}{2} \mathcal{A}^{-1}(k) \mathcal{B}(k) .
$$

\section{II.D. Controller Construction}

The control $u(k)$ is given by the strictly proper time-series controller of order $n_{\mathrm{c}}$ given by

$$
u(k)=\sum_{i=1}^{n_{\mathrm{c}}} M_{i}(k) u(k-i)+\sum_{i=1}^{n_{\mathrm{c}}} N_{i}(k) y(k-i),
$$

where, for all $i=1, \ldots, n_{\mathrm{c}}, M_{i}(k) \in \mathbb{R}^{l_{u} \times l_{u}}$ and $N_{i}(k) \in \mathbb{R}^{l_{u} \times l_{y}}$. The control (27) can be expressed as

$$
u(k)=\theta(k) \phi(k-1),
$$

where

$$
\theta(k) \triangleq\left[M_{1}(k) \cdots M_{n_{\mathrm{c}}}(k) N_{1}(k) \cdots N_{n_{\mathrm{c}}}(k)\right] \in \mathbb{R}^{l_{u} \times n_{\mathrm{c}}\left(l_{u}+l_{z}\right)}
$$


and

$$
\phi(k-1) \triangleq\left[\begin{array}{c}
u(k-1) \\
\vdots \\
u\left(k-n_{\mathrm{c}}\right) \\
y(k-1) \\
\vdots \\
y\left(k-n_{\mathrm{c}}\right)
\end{array}\right] \in \mathbb{R}^{n_{\mathrm{c}}\left(l_{u}+l_{y}\right)}
$$

\section{II.E. Recursive Least Squares Update of $\theta(k)$}

Next, let $d$ be a positive integer such that $\tilde{U}(k-1)$ contains $u(k-d)$ and define the cumulative cost function

$$
J_{\mathrm{R}}(\theta, k) \triangleq \sum_{i=d+1}^{k} \lambda^{k-i}\left\|\phi^{\mathrm{T}}(i-d-1) \theta^{\mathrm{T}}(k)-\hat{u}^{\mathrm{T}}(i-d)\right\|^{2}+\lambda^{k}\left(\theta(k)-\theta_{0}\right) P_{0}^{-1}\left(\theta(k)-\theta_{0}\right)^{\mathrm{T}},
$$

where $\|\cdot\|$ is the Euclidean norm, and $\lambda \in(0,1]$ is the forgetting factor. Minimizing (31) yields

$$
\begin{aligned}
\theta^{\mathrm{T}}(k)= & \theta^{\mathrm{T}}(k-1)+\beta(k) P(k-1) \phi(k-d-1) \cdot\left[\phi^{\mathrm{T}}(k-d) P(k-1) \phi(k-d-1)+\lambda(k)\right]^{-1} \\
& \cdot\left[\phi^{\mathrm{T}}(k-d-1) \theta^{\mathrm{T}}(k-1)-\hat{u}^{\mathrm{T}}(k-d)\right],
\end{aligned}
$$

where $\beta(k)$ is either zero or one. The error covariance is updated by

$$
\begin{aligned}
P(k)= & \beta(k) \lambda^{-1} P(k-1)+[1-\beta(k)] P(k-1)-\beta(k) \lambda^{-1} P(k-1) \phi(k-d-1) \\
& \cdot\left[\phi^{\mathrm{T}}(k-d-1) P(k-1) \phi(k-d)+\lambda\right]^{-1} \cdot \phi^{\mathrm{T}}(k-d-1) P(k-1) .
\end{aligned}
$$

We initialize the error covariance matrix as $P(0)=\alpha I_{3 n_{c}}$, where $\alpha>0$. Note that when $\beta(k)=0, \theta(k)=$ $\theta(k-1)$ and $P(k)=P(k-1)$. Therefore, setting $\beta(k)=0$ switches off the controller adaptation, and thus freezes the control gains. When $\beta(k)=1$, the controller is allowed to adapt.

\section{Model and Controller Setup}

In this section, we describe how the aerodynamic stability derivatives within GTM are accessed, and we present the RCAC architecture.

The GTM has an extensive lookup table of aerodynamic coefficients, which are programmed in Simulink as functions of angle of attack and side slip angle. These coefficients represent normalized forces and moments that can be acquired from either computational fluid dynamics software or empirically from an extensive wind tunnel experiment. Although these coefficients are related to the stability derivatives, individual stability derivatives are not specified. An illustration of this database is shown in Figure 1(a) for the force coefficient along the aircraft body $z$-axis, with the body-axis defined in Figure 1(b). The aerodynamic coefficients that are modified in this paper to illustrate $\mathrm{RCAC}$ are denoted by $C_{\mathrm{F}(\cdot)}$ for a force coefficient and $C_{\mathrm{M}(\cdot)}$ for a moment coefficient in the $(\cdot)$-axis about the body frame.

For this study, we focus on the ability of RCAC to control the aircraft to maintain straight and level flight despite unknown time varying lift and drag aerodynamics. To achieve this objective, RCAC is set up as shown in Figure 2, where the components of $r \in \mathbb{R}^{4}$ represent altitude, airspeed, roll angle, and heading angle commands. Since the simulation is initialized at a given trim state, the reference commands are just these trim conditions. Feedback is utilized from the model to define the components of $z \in \mathbb{R}^{4}$ as the respective performance error signals of altitude, airspeed, roll angle, and heading angle. The output of $\mathrm{RCAC}$ is $\delta_{\mathrm{LT}}, \delta_{\mathrm{RT}}, \delta_{e}, \delta_{a}, \delta_{r} \in \mathbb{R}$, which are the commands to the left and right engines, elevator, aileron, and rudder, respectively. Also, Figure 2 shows the RCAC setup and how it is integrated with GTM. It is important to note that RCAC is not given any knowledge of when or how the aerodynamic coefficients will be changing. This implies that to compensate for these changes, RCAC must apply real-time adaptation and utilize the available control authority. 


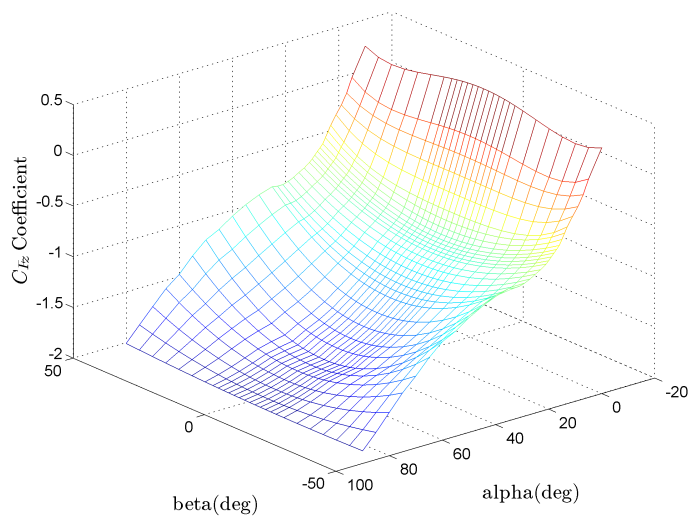

(a) Body $z$-force aerodynamic coefficient mesh as a function of the angle of attack $(\alpha)$ and side slip angle $(\beta)$

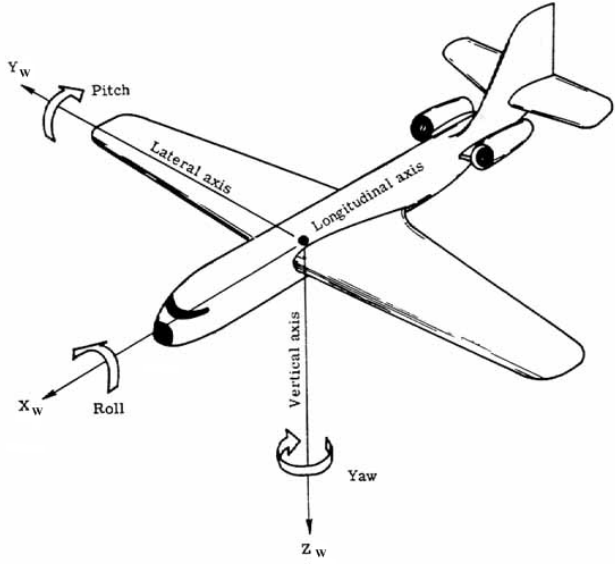

(b) Aircraft body coordinate system

Figure 1. A sample of the GTM aerodynamic data base (a) and an illustration of the aircraft body frame (b)

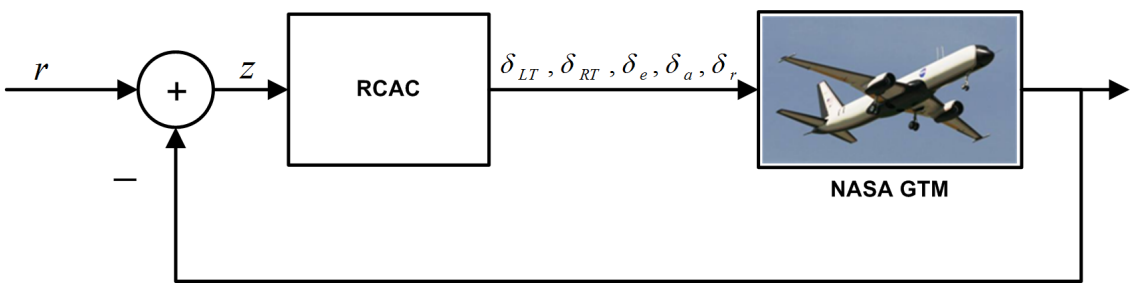

Figure 2. RCAC architecture

\section{Results}

This section presents results based on the control architecture outlined in Section III. As noted above, each example is commanded to maintain the initial trim altitude and airspeed. Since the goal is to maintain straight and level flight despite unknown time varying lift and drag forces, the force coefficients modifications are applied along the aircraft body $x$ - and $z$-axes. For each case, RCAC is tasked to maintain a desired altitude, heading, roll angle, and airspeed while the aerodynamic coefficients are modified at time $t=500$ sec. These changes range from sudden transformations as in Example IV.1 and IV.2 to a linearly time varying transformation in Example IV.3.

In all of the examples below, RCAC utilizes a single tuning with the parameters

$$
\begin{aligned}
n_{c} & =10, \\
P(0)= & 10 I_{\left(l_{r}+l_{u}\right) n_{c}}, \\
\tilde{\mathcal{H}}= & {\left[\begin{array}{lllll}
H_{1} & H_{2} & H_{3} & H_{4} & H_{5}
\end{array}\right], } \\
R_{Z}= & {\left[\begin{array}{cccc}
R_{z_{1}} & \mathbf{0} & \mathbf{0} & \mathbf{0} \\
\mathbf{0} & R_{z_{2}} & \mathbf{0} & \mathbf{0} \\
\mathbf{0} & \mathbf{0} & R_{z_{3}} & \mathbf{0} \\
\mathbf{0} & \mathbf{0} & \mathbf{0} & R_{z_{4}}
\end{array}\right], } \\
R_{U}= & {\left[\begin{array}{ccccc}
R_{u_{1}} & 0 & 0 & 0 & 0 \\
0 & R_{u_{2}} & 0 & 0 & 0 \\
0 & 0 & R_{u_{3}} & 0 & 0 \\
0 & 0 & 0 & R_{u_{4}} & 0 \\
0 & 0 & 0 & 0 & R_{u_{5}}
\end{array}\right], }
\end{aligned}
$$




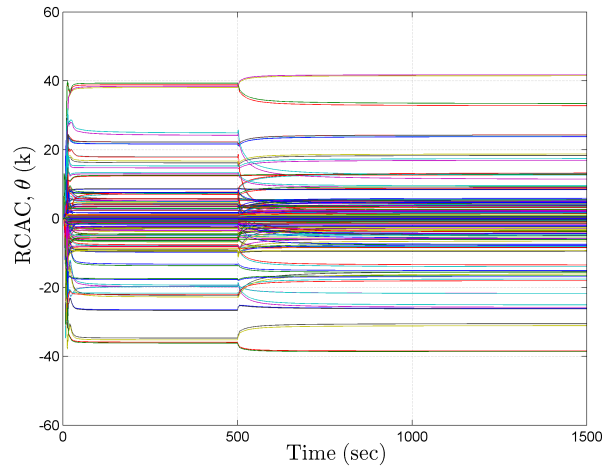

(a)

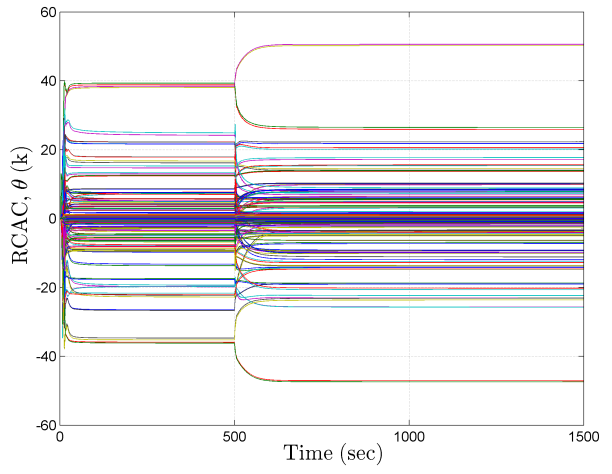

(b)

Figure 3. The evolution of the RCAC gains for Example IV.1. (a) shows the gains for a $100 \%$ increase in drag, while (b) shows the gains for a $200 \%$ drag increase. The modified aerodynamic coefficient $C_{\mathrm{F}_{\mathrm{x}}}$ is introduced into the simulation at $t=500$ sec.

where

$$
R_{z_{1}}=R_{z_{2}}=R_{z_{3}}=R_{z_{4}}=\left[\begin{array}{cccc}
1.5 & 0 & 0 & 0 \\
0 & 1.0 & 0 & 0 \\
0 & 0 & 1.0 & 0 \\
0 & 0 & 0 & 30
\end{array}\right]
$$

and

$$
\left[\begin{array}{lllll}
R_{u_{1}} & R_{u_{2}} & R_{u_{3}} & R_{u_{4}} & R_{u_{5}}
\end{array}\right]=\left[\begin{array}{lllll}
0.002 & 0.002 & 0.70 & 0.70 & 0.07
\end{array}\right] .
$$

The Markov parameters in (34) are calculated based on a single linearized version of GTM with the appropriate states modeled in the system.

Example IV.1. Sudden Increase in Drag

Consider the case where the drag abruptly increases by an unknown scale factor. In this example, only the coefficient $C_{\mathrm{F}_{\mathrm{X}}}$ is modified. For this case, the coefficient is modeled as

$$
C_{\mathrm{Fx}}=\left(1+\frac{\eta_{\mathrm{Fx}}}{100}\right) C_{\mathrm{Fx} 0}
$$

where $C_{\mathrm{F}_{\mathrm{x} 0}}$ is the $x$-axis force coefficient calculated from the aerodynamic database, $\eta_{\mathrm{F}_{\mathrm{x}}}$ is the percentage value increase from $C_{\mathrm{Fx} 0}$, and $C_{\mathrm{Fx}}$ is the modified body $x$-axis force coefficient utilized in the simulation. The aerodynamic change occurs in the simulation at $t=500 \mathrm{sec}$.

Figure 3 shows the evolution of the RCAC gains, where (a) is the result of increasing the drag coefficient by $100 \%$ and (b) is due to an increase of $200 \%$. Note that RCAC has no prior knowledge of the abrupt change in drag. Figure 4 shows the results for a $0 \%, 100 \%$, and $200 \%$ increase in drag. As shown in Figures $4(\mathrm{a})$ and 4(b), the performance variables of altitude and airspeed are affected at time $500 \mathrm{sec}$ but are compensated for and remain close to the desired performance values. Both Figures 4(c) and 4(d) are the control variables used to compensate for this change in dynamics. As shown, the elevator deflects appropriately due to the changes in the aerodynamic coefficient in order to maintain a suitable angle of attack (Figure 4(e)) for generating the lift necessary for altitude performance. The thrust increases to compensate for the abrupt increase in $C_{\mathrm{Fx}}$.

Example IV.2. Sudden Decrease in Lift

As in Example IV.1, we consider a sudden scale factor change at time $500 \mathrm{sec}$ but this time affecting the lift. For this case, the coefficient is modeled as

$$
C_{\mathrm{Fz}}=\left(1-\frac{\eta_{\mathrm{F} z}}{100}\right) C_{\mathrm{F} 0}
$$




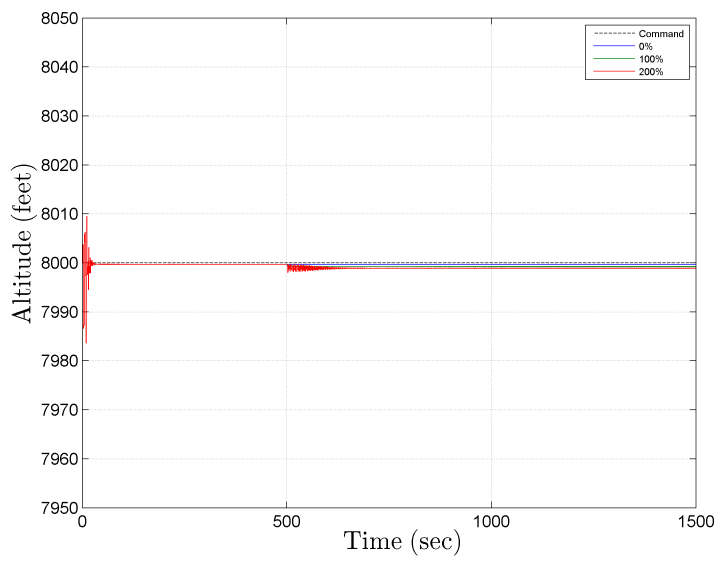

(a)

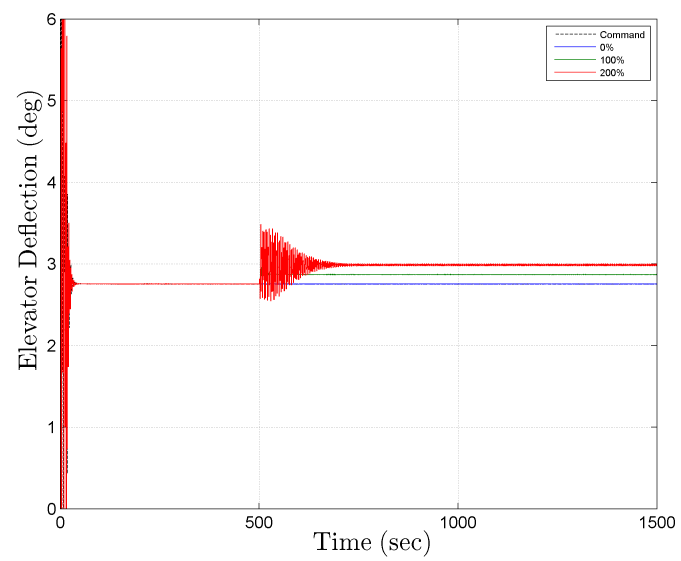

(c)

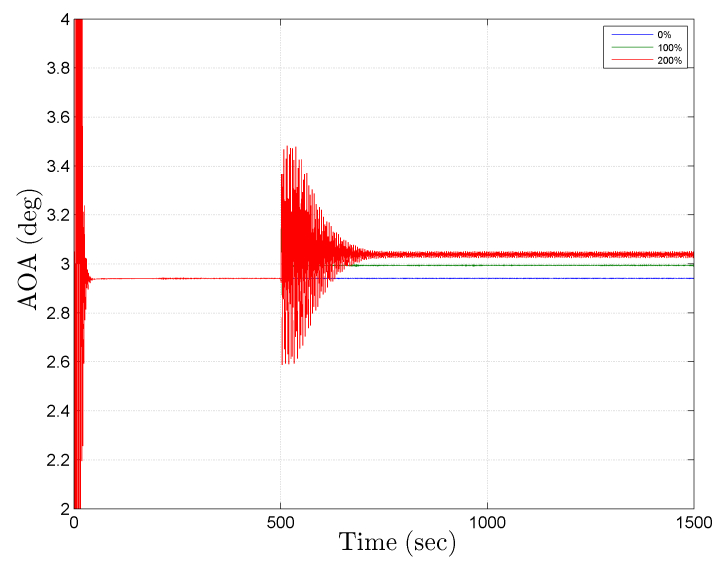

(e)

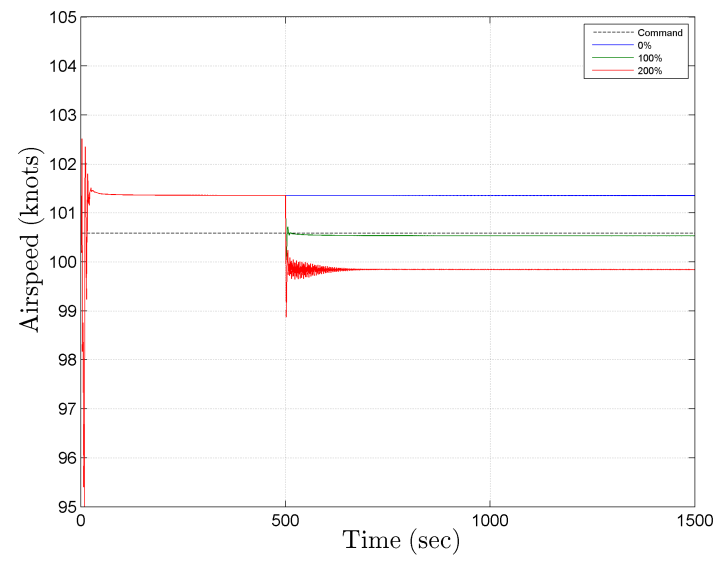

(b)

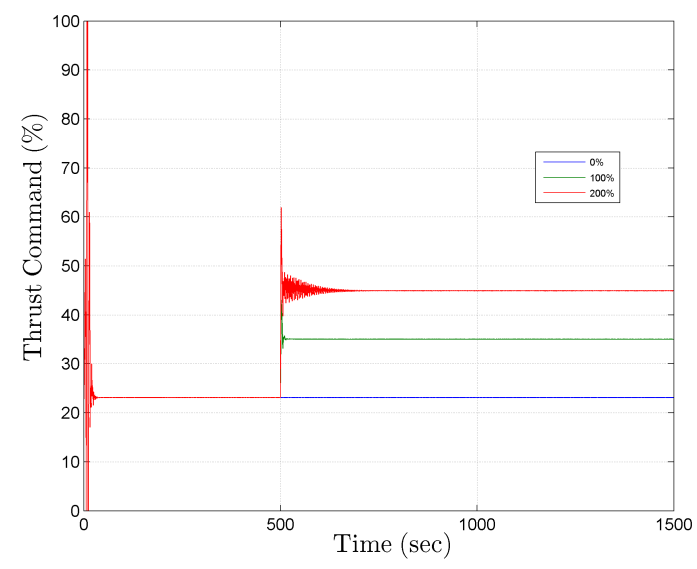

(d)
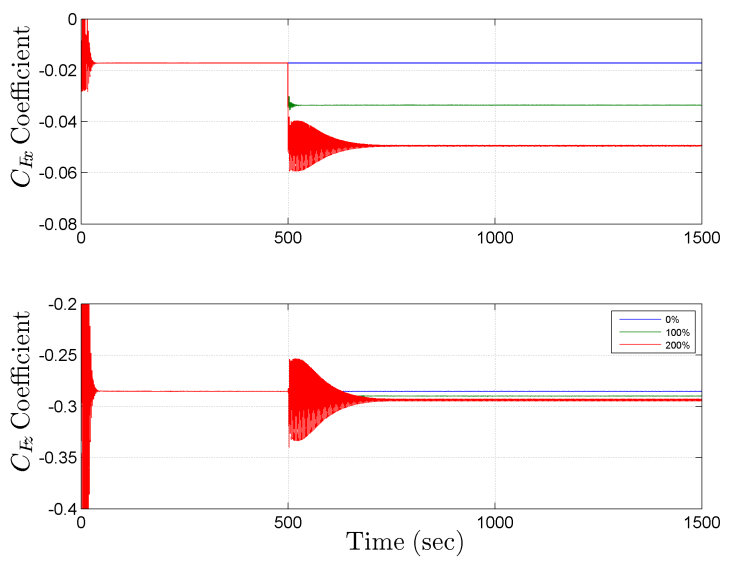

(f)

Figure 4. Example IV.1. The performance variable altitude (a) and airspeed (b) are affected at time 500 sec, RCAC recovers with the control authority of the elevator (c) and thrust (d) react appropriately to reject the abrupt increase in drag. (e) shows the angle of attack $(\alpha)$ adjustment for the decrease in lift, and (f) shows the aerodynamic coefficients. Note that the coefficient $C_{\mathrm{F}_{\mathrm{x}}}$ illustrates the increases in drag while the lift coefficient $C_{\mathrm{F}_{\mathrm{z}}}$ is only slightly modified due to dynamic coupling. 


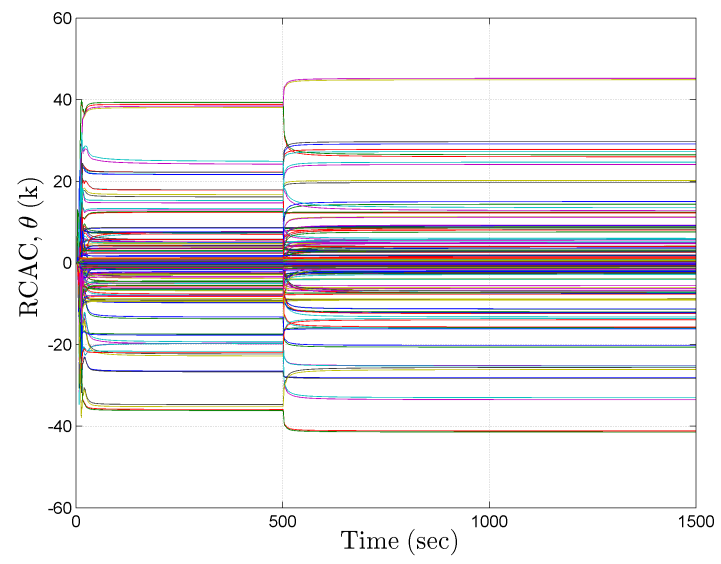

(a)

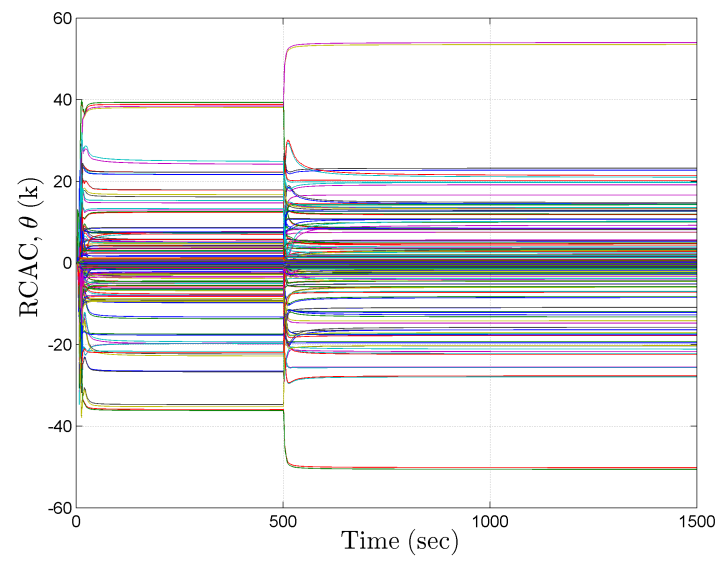

(b)

Figure 5. Evolution of the gains for Example IV.2. (a) shows the gains of RCAC for a $20 \%$ decrease in lift, while (b) shows the gains for a $40 \%$ decrease in lift. Note that the gains evolve from one steady-state value for $t<500$ sec to another for $t>500$ sec due to the abrupt decrease in lift.

where $C_{\mathrm{F}_{\mathrm{z} 0}}$ is the $z$-axis force coefficient calculated from the aerodynamic database, $\eta_{\mathrm{F}_{\mathrm{z}}}$ is the percentage decrease from $C_{\mathrm{F}_{\mathrm{z} 0}}$, and $C_{\mathrm{F}_{\mathrm{z}}}$ is the modified body $z$-axis force coefficient utilized in the aircraft equations of motion.

Figure 5 shows the evolution of the RCAC gains for a 20\% decrease in lift shown in Figure 5(a) and 40\% decrease in lift as shown in Figure 5(b). Note that the gains evolve more rapidly in the $40 \%$ case than the $20 \%$ case due to the difference in magnitude of the change in the aerodynamic coefficient.

Figure 6 shows the results due to a 0\%,20\%, and 40\% decrease in lift. As shown in Figures 6(a) and 6 (b) the performance variables (altitude and airspeed) are affected at time $500 \mathrm{sec}$ but are compensated for and remain close to the desired performance. Both Figures 6(c) and 6(d) depict the control authority used to compensate for this abrupt change in lift. As shown, the elevator deflects in the correct direction with suitable magnitude in order to increase the angle of attack (Figure 6(e)) and therefore maintain the lift necessary for the altitude, while the thrust is decreased slightly to maintain airspeed. By modifying $R_{z}$ in the RCAC algorithm it is possible to weigh the altitude performance relative to the airspeed due to the aircraft's inabitility to produce the lift necessary to maintain altitude at a given airspeed without stalling.

Example IV.3. Icing Example

To emulate the effect of icing on the lifting surfaces of the aircraft, the aerodynamic force coefficients $C_{\mathrm{Fx}}$ and $C_{\mathrm{Fz}_{\mathrm{z}}}$ are modified. In particular, we model icing as the degradation of lift produced by the wings and an increase in drag. The aerodynamic coefficients are modeled as

$$
C_{\mathrm{F}(\cdot)}= \begin{cases}C_{\mathrm{F}(\cdot)_{0}}, & t<500 \mathrm{sec}, \\ C_{\alpha \mathrm{F}(\cdot)}(t-500)+C_{\mathrm{F}(\cdot)_{0}}, & 500 \mathrm{sec} \leq t<1000 \mathrm{sec}, \\ 500 C_{\alpha \mathrm{F}(\cdot)}+C_{\mathrm{F}(\cdot)_{0}}, & t \geq 1000 \mathrm{sec},\end{cases}
$$

where $(\cdot)$ is the desired body axis label $(x, y, z), C_{\mathrm{F}(\cdot)_{0}}$ is the axis force coefficient calculated from the aerodynamic database, and $C_{\alpha \mathrm{F}(\cdot)}$ is the slope of the coefficient modification governed by

$$
C_{\alpha \mathrm{F}(\cdot)}=\frac{\eta_{\mathrm{F}(\cdot)}}{100} \frac{\Lambda_{\mathrm{F}(\cdot)}}{500}
$$

where $\Lambda_{\mathrm{F}(\cdot)}$ is the axis force coefficient from the database at $t=500 \mathrm{sec}$ and $\eta_{\mathrm{F}(\cdot)}$ is the percent increase/decrease from $\Lambda_{\mathrm{F}(\cdot)}$. For this example, these constants are

$$
\begin{array}{ll}
\Lambda_{\mathrm{F} x}=-0.01676, & \eta_{\mathrm{F} x}=700 \\
\Lambda_{\mathrm{F} z}=-0.29051, & \eta_{\mathrm{F} z}=-30 .
\end{array}
$$




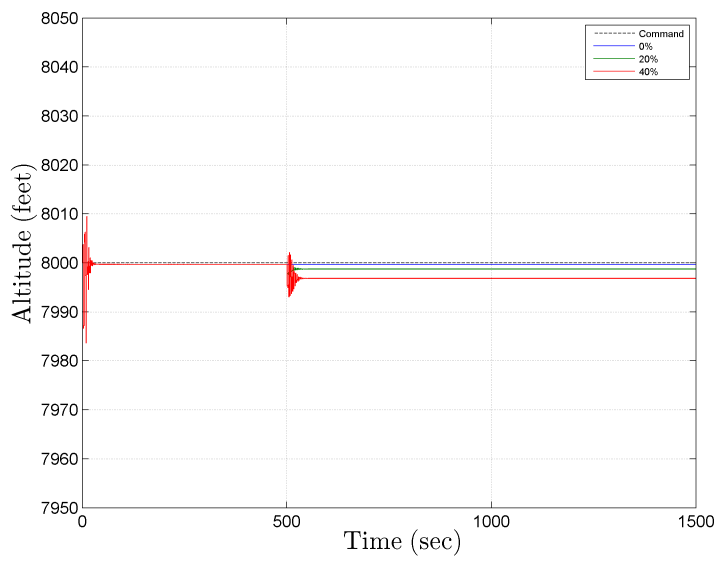

(a)

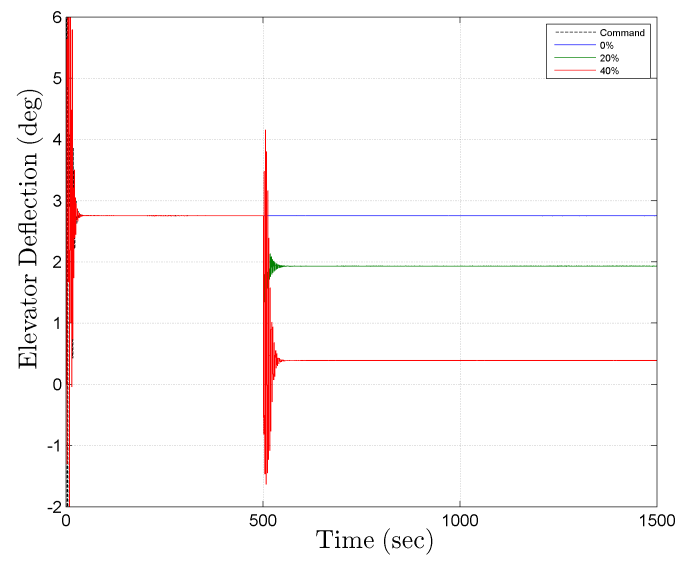

(c)

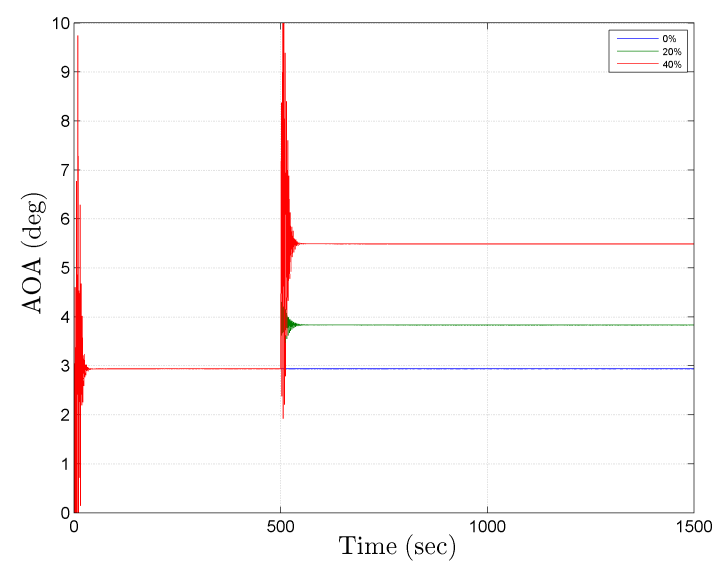

(e)

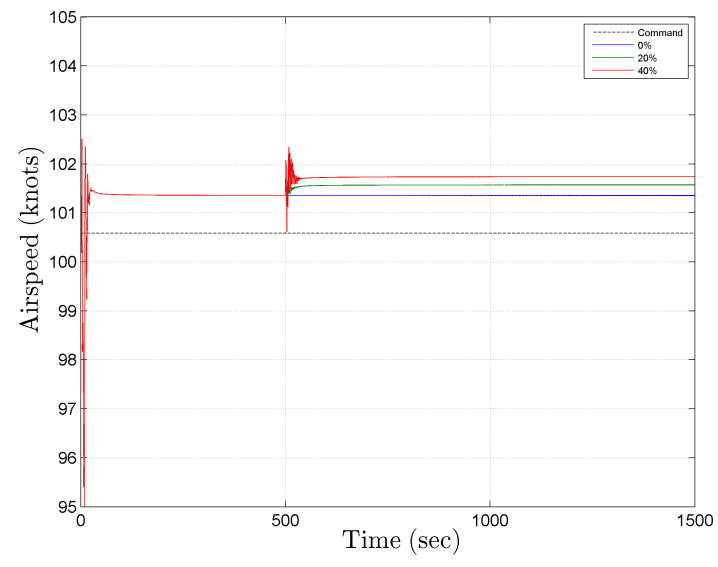

(b)

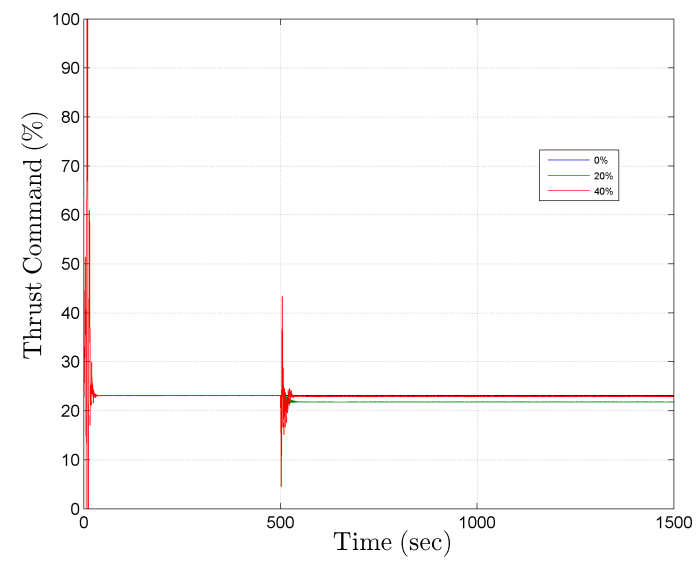

(d)
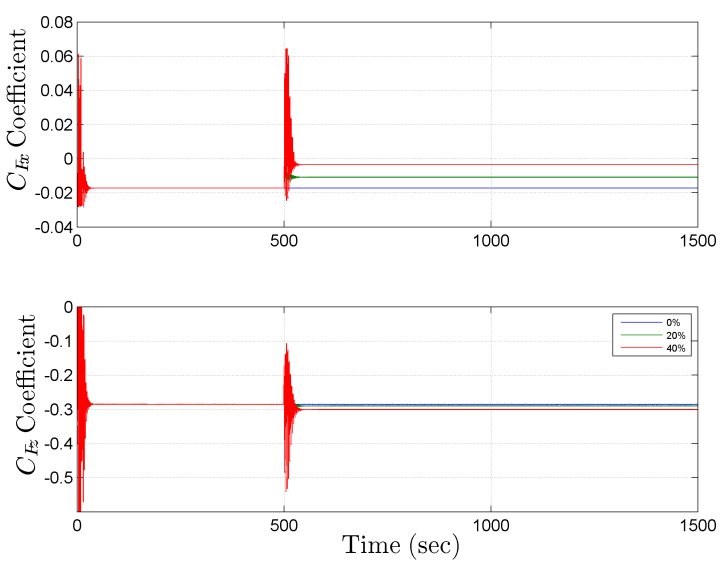

(f)

Figure 6. Example IV.2. These results show how the performance variables of altitude (a) and airspeed (b) are affected at time 500 sec but recovers with control authority from the elevator (c) and thrust (d). (e) shows the angle of attack $(\alpha)$ adjustment for the decrease in lift and (f) shows the aerodynamic coefficients. Note that $C_{\mathrm{F}}$, remains unchanged past 500 sec to maintain the appropriate lift in order to hold the desired altitude performance, while $C_{\mathrm{F}} \mathrm{decreases}$ resulting in less thrust necessary to maintain airspeed and steady level flight 


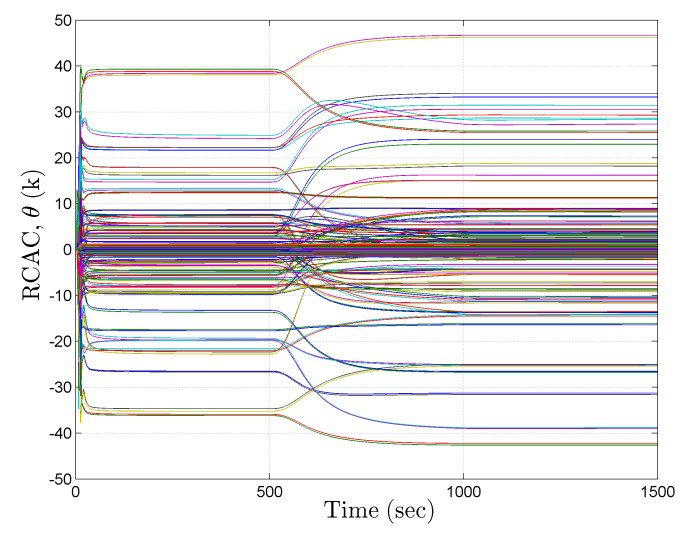

Figure 7. Evolution of the RCAC gains for Example IV.3. Note that the controller gain evolve considerably over 500 sec $<t<1000$ sec to adapt to the unknown time-varying aerodynamic coefficients.

Note that at time $500 \mathrm{sec}, C_{F x}$ is linearly increased by $700 \%$, while $C_{F z}$ is linearly decreased by $30 \%$ in 500 sec as dictated by (39).

Figure 7 shows the evolution of the gains of the RCAC algorithm. Note that at $t<500$ sec the gains converge to a steady value, but during $500 \mathrm{sec}<t<1000 \mathrm{sec}$, the gains evolve to compensate for the aerodynamic coefficient modifications. For $t>1000 \mathrm{sec}$, the gains again converge to a steady-state value since the coefficients are no longer changing.

Figures 7 and 8 show the results for the icing example. Figure 8(a) and Figure 8(b) show how the performance variables of altitude and airspeed are maintained near the desired reference signal. The control authority needed to maintain the desired states are shown in Figure 8(c) and Figure 8(d). Note that the thrust increases to compensate for the increase in drag, and the elevator decreases to increase the angle of attack shown in Figure 8(e). Figure 8(f) illustrates the aerodynamic coefficients before and after they are modified. Note that $C_{\mathrm{F}(\cdot)_{0}}$ is the coefficient from the database prior to modification.

\section{Conclusions}

In this paper we applied retrospective cost adaptive control (RCAC) to the NASA GTM model under unanticipated and unknown changes to the aerodynamics of the aircraft. Specifically, we used a single RCAC block within the simulation that controls the aircraft to a desired steady level flight by commanding five actuation channels (left and right engines, aileron, elevator, and rudder). The goal is to examine the evolution of the RCAC controller gains in response to changes in the aerodynamic coefficients from the linearized aircraft dynamics. To show this, simulations in the Simulink GTM were run, all with the desire for the aircraft to fly in a straight and level flight configuration. At a certain time (unknown to RCAC) the aerodynamic coefficients changed. Presented were three different aerodynamic parameter modifications, including an icing example, a sudden increase in drag, and a sudden decrease in lift. For all three examples, only a single tuning was used for each RCAC block.

Results showed that RCAC is able to adapt its gains in order to compensate for the unknown time-varying aerodynamic perturbations while maintaining the desired performance. The icing example showed that both the elevator and engines were used to compensate for an increase of $700 \%$ drag and decrease of $30 \%$ in lift production over a $500 \mathrm{sec}$ interval. In the drag example, RCAC overcame a increase in the aerodynamic drag coefficient by over $200 \%$ by increasing the engine thrust and using minimal elevator deflections. The gains evolved substantially to compensate for the amount of drag induced. Finally, we considered an example where the lift decreased by $40 \%$. Given the constraints imposed by the performance variable on the airspeed, RCAC used the elevator to increase the angle of attack to maintain altitude. Future research focus on additional aerodynamic stability derivative changes as well as a method for relaxing constraints to maintain safe level flight. 


\section{Acknowledgment}

This work was supported in part by the National Aeronautics and Space Administration under Cooperative Agreement NNX12AM54A.

\section{References}

${ }^{1}$ R. Bailey and T. Jordan. NASA Langleys airstar testbed: A subscale flight test capability for flight dynamics and control system experiments. In AIAA Guidance, Navigation, and Control Conference and Exhibit, Honolulu, HI, Aug 2008.

${ }^{2}$ M. Blanke. Diagnosis and Fault-Tolerant Control. Engineering online library. Springer, 2003.

${ }^{3}$ A. M. D'Amato, E. D. Sumer, and D. S. Bernstein. Frequency-domain stability analysis of retrospective-cost adaptive control for systems with unknown nonminimum-phase zeros. In Decision and Control and European Control Conference (CDCECC), 2011 50th IEEE Conference on, pages 1098 -1103, Dec. 2011.

${ }^{4}$ A. M. D'Amato, E. D. Sumer, K. Mitchell, A. Morozov, J. B. Hoagg, and D. S Bernstein. Adaptive output feedback control of the NASA GTM model with unknown nonminimum-phase zeros. In AIAA Guidance, Navigation, and Control Conference, Portland, OR, Aug 2011.

${ }^{5}$ D. Dasgupta, K. Krishna-Kumar, D. Wong, and M. Berry. Negative selection algorithm for aircraft fault detection. In Nicosia, Cutello, Bentley, and Timmis, editors, Artificial Immune Systems, volume 3239 of Lecture Notes in Computer Science, pages 1-13. Springer Berlin Heidelberg, 2004.

${ }^{6}$ J. Gertler. Fault Detection and Diagnosis in Engineering Systems. Marcel Dekker, 1998.

${ }^{7}$ J. B. Hoagg and D. S. Bernstein. Retrospective cost model reference adaptive control for nonminimum-phase systems. Journal of Guidance, Control, and Dynamics, 35(6):1767-1786, Oct 2012.

${ }^{8}$ J. B. Hoagg, A. M. Santillo, and D. S. Bernstein. Discrete-time adaptive command following and disturbance rejection with unknown exogenous dynamics. Automatic Control, IEEE Transactions on, 53(4):912 -928, May 2008.

${ }^{9}$ R. Isermann. Fault-Diagnosis Systems: An Introduction from Fault Detection to Fault Tolerance. Springer-Verlag Berlin Heidelberg, 2006.

${ }^{10}$ A. Murch. A flight control system architecture for the NASA AirSTAR flight test infrastructure. In AIAA Guidance, Navigation, and Control Conference and Exhibit, Honolulu, HI, Aug 2008.

${ }^{11}$ J. Roskam. Airplane Flight Dynamics and Automatic Flight Controls. Number pt. 2 in Airplane Flight Dynamics and Automatic Flight Controls. Darcorporation, 2003.

12 A. M. Santillo and D. S. Bernstein. Adaptive control based on retrospective cost optimization. Journal of Guidance, Control, and Dynamics, 33(2):289-304, Feb 2010.

${ }^{13}$ R. Venugopal and D. S. Bernstein. Adaptive disturbance rejection using ARMARKOV system representations. In Decision and Control, 1997., Proceedings of the 36th IEEE Conference on, pages 1884-1889 vol. 2, Dec 1997. 


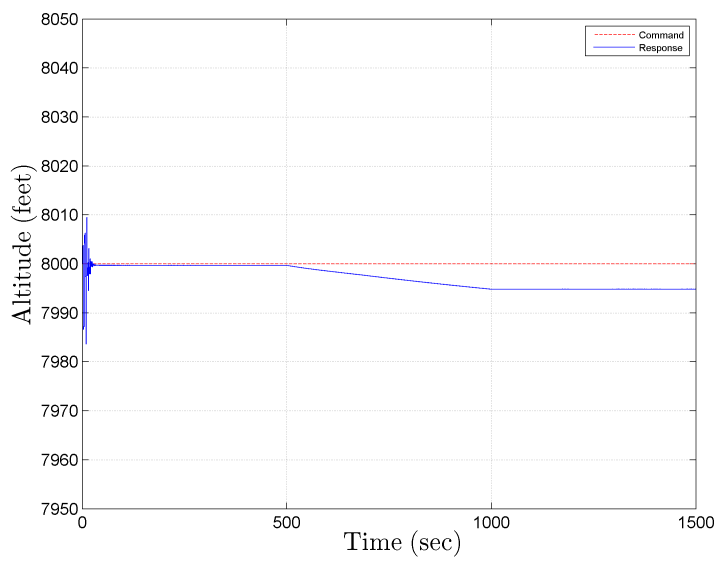

(a)

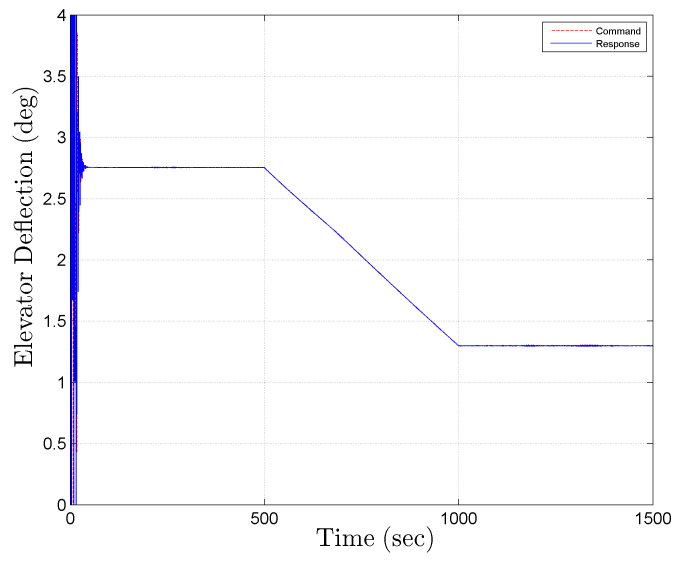

(c)

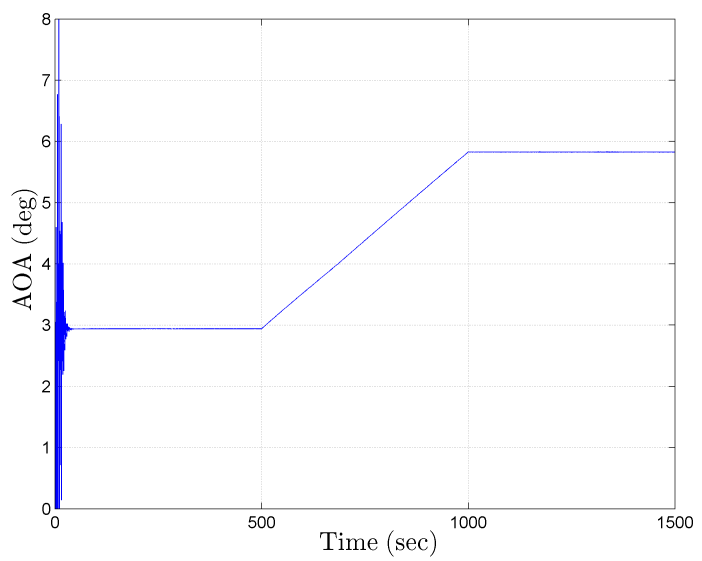

(e)

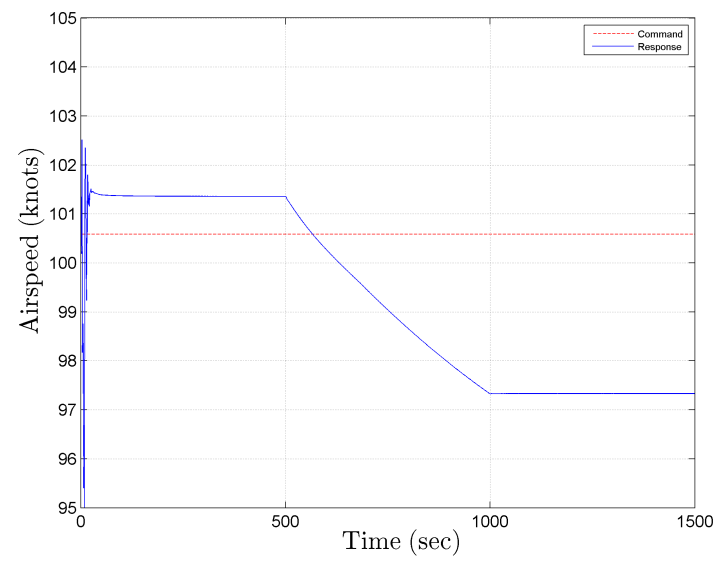

(b)

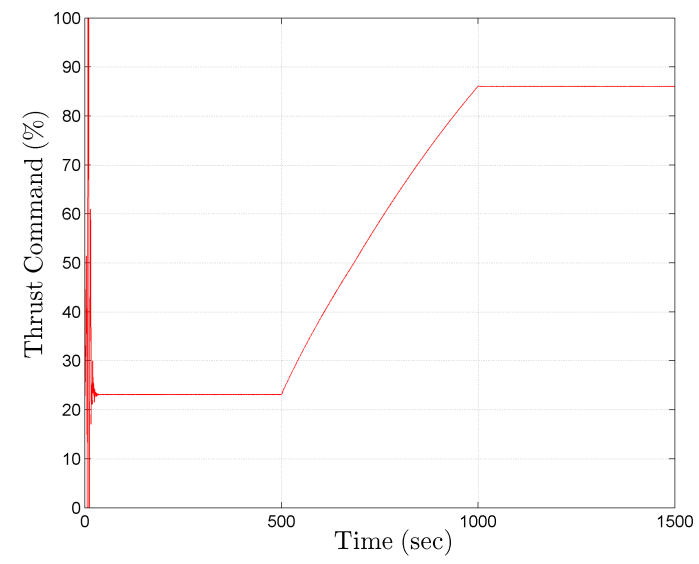

(d)
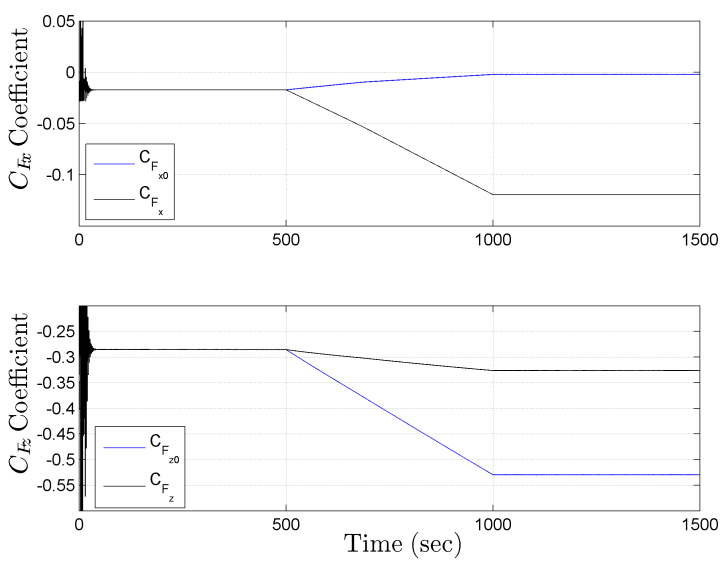

(f)

Figure 8. Example IV.3. The performance variable of altitude (a) and airspeed (b) with control authority of the elevator (c) and engine thrust (d) follow the altitude and airspeed commands well despite changes in the aerodynamic coefficients at 500 sec. (e) shows the angle of attack $(\alpha)$ adjustment for the decrease in lift, and (f) shows the aerodynamic coefficients. Note that unlike $C_{\mathrm{F}_{\mathrm{x}}}, C_{\mathrm{F}_{\mathrm{z}}}$ does not vary greatly after 500 sec. This is because the elevator deflects to increase $\alpha$ to maintain the same lift coefficient for the desired airspeed, while the thrust is used to compensate for the increase in drag. 